\title{
REGENERAÇÃO DE ARGILAS DA INDÚSTRIA DE ALIMENTOS POR TRATAMENTO TÉRMICO/QUÍMICO
}

\author{
C. M. S. NASCIMENTO ${ }^{1}$, L.J. FERNANDES ${ }^{2}$, R.S. MELO ${ }^{2}$, E.C. SANTOS ${ }^{3}$, J.M. FERREIRA ${ }^{2,4}$, \\ M.A MOTTA SOBRINHO ${ }^{1,2}$ \\ ${ }^{1}$ Universidade Federal de Pernambuco, Programa de Pós-Graduação em Engenharia Civil \\ ${ }^{2}$ Universidade Federal de Pernambuco, Departamento de Engenharia Química \\ ${ }^{3}$ Instituto Tecnológico de Pernambuco, Departamento de Engenharia Química \\ ${ }^{3}$ Universidade Federal da Paraíba, Departamento de Tecnologia Sucroalcooleira \\ E-mail para contato: mottas@ufpe.br
}

\begin{abstract}
RESUMO - A clarificação é uma etapa essencial no refino de óleos vegetais. O descoramento ou purificação de óleos comestíveis envolve a remoção de componentes coloridos, traços de metais, fosfolipídeos, sabões e produtos de oxidação como peróxidos. Geralmente, argilas naturais ou comerciais ativadas são empregadas industrialmente como adsorventes no descoramento de óleo vegetal. $\mathrm{O}$ adsorvente pode ser recuperado através de tratamentos químicos com ácidos ou térmicos. O objetivo deste trabalho foi avaliar a regeneração destas argilas através de um tratamento térmico e de um tratamento químico. Foram coletadas amostras da Bunge, utilizadas na purificação de óleo vegetal. Foi realizado um planejamento experimental para otimizar o tratamento térmico e foram avaliados cinco solventes diferentes para extração do óleo. Os resultados indicaram que a o processo deverá ser conduzido com um pré-tratamento na estufa a $105{ }^{\circ} \mathrm{C}$, seguido de um tratamento na mufla a $1000^{\circ} \mathrm{C}$ por 30 minutos. Em relação aos solventes, o hexano apresentou maior eficiência.
\end{abstract}

\section{INTRODUÇÃO}

A produção mundial de óleo vegetal, na safra 2013/14 foi de 168,98 milhões de metros cúbicos, dos quais o óleo de palma corresponde a $34 \%$ e o de soja a $26 \%$. Ressalta-se ainda que nos últimos 5 anos a produção aumentou 19,54\% (USDA, 2014). No Brasil, o óleo de soja é o que predomina, com uma produção anula de 7,1 milhões de toneladas, das quais, 5,8 ton abastecem o mercado interno e 1,3 ton seguem para exportação, segundo previsão da Associação Brasileira das Indústrias de Óleos Vegetais para o ano de 2014 (ABIOVE, 2014).

O óleo vegetal bruto, geralmente, não pode ser consumido diretamente, pois possui substâncias que podem alterar suas características, podendo provocar danos à saúde humana. Todavia, deve-se ressaltar que existem algumas exceções como o óleo de dendê e o óleo de oliva, os quais são consumidos diretamente após a extração. O processo de tratamento (refino) do óleo envolve diversas operações e reações, tornando-o um produto comercial (Patterson, 1992; O’Brien et al., 2000). Dentre as características que o óleo deve possuir para o consumo humano destacam-se a cor clara, o sabor 


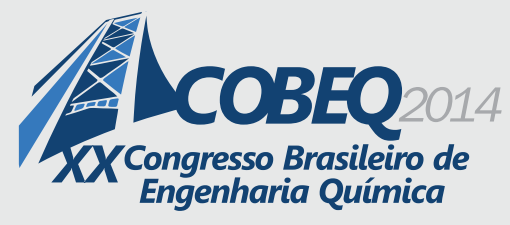

suave e a estabilidade oxidativa (González-Tovar et al., 2005).

O refino do óleo vegetal se inicia com a degomagem, na qual são removidos fosfotídeos, proteínas e substância coloidais após o tratamento por centrifugação. Segue-se então a neutralização para a remoção dos ácidos graxos livres, proteínas e outros contaminantes. Finalmente, o óleo vai para a etapa de clareamento, para reduzir/eliminar as impurezas (metais pesados, aldeídos, fosfatos, etc.) e substâncias que conferem cor (pigmentos) ao óleo. Várias destas substâncias podem atuar promover reações indesejáveis que deterioram a qualidade do óleo, como a reação de oxidação. Estas substâncias também podem interferir no processo de tratamento, como é o caso presença da clorofila, que mesmo em concentrações baixas, aparecem visualmente após a etapa de hidrogenação (Sonntag et al., 1979; Güller e Tunç, 1992; Lima, 2003).

Cardello et al., (1995) afirmaram que a clarificação é uma etapa fundamental no processo de beneficiamento do óleo comestível, pois assegura a estabilidade e qualidade do produto final. Este processo elimina os elementos indesejáveis, tais como os corantes (a clorofila-a e betacaroteno) e outros resíduos (resíduos de sabão, traços de metais pesados, etc.), contidos no óleo bruto (Sabah et al., 2007). Ressalta-se que o objetivo inicial da clarificação é a remoção de corantes, todavia com a prática percebeu-se que compostos indesejáveis, citados anteriormente, também eram removidos.

Teixeira-Neto \& Teixeira-Neto (2009) afirmam que quando a argila bentonita é utilizada para a clarificação de óleos comestíveis, realiza-se uma ativação da mesma com ácido clorídrico ou sulfúrico. O tratamento ácido promove alterações na estrutura da argila (aumento da porosidade e da acidez), além de conferir-lhe maior estabilidade térmica.

Silva et al. (2013) verificaram que a adsorção de fósforo e beta-caroteno seguiram os modelos de Freundlich e Langmuir, respectivamente, e que o aumento da temperatura favorece a adsorção, indicando uma possível adsorção física. Sabah et al. (2007) também verificaram o mesmo comportamento na sepiolita para o beta caroteno. Todavia, Garofalo (2011) cita que, na maioria dos casos, a descoloração dos óleos vegetais por adsorção ocorre por adsorção física. Desta forma, para se promover a dessorção destes materiais deverá se ter uma elevada temperatura e agitação intensa. Ele ressalta ainda a importância na escolha do solvente, que deve ter afinidade com os compostos a serem removidos deste adsorvente.

Meziti e Boukerroui (2011) estudaram a dessorção do óleo da terra de clarificação. Eles trataram inicialmente o material com uma solução de cloreto de amônio $(3 \mathrm{M})$, seguido de um tratamento térmico na mufla a $400^{\circ} \mathrm{C}$ durante uma hora e finalizando com um tratamento químico (uma lavagem a frio com $\mathrm{HCl} 1 \mathrm{M}$ ). Eles observaram que o tratamento não alterou a estrutura da montmorolonita e que o cloreto de amônia sob efeito da temperatura aumentou a micro porosidade do material regenerado.

Desta forma, este trabalho tem por objetivo a recuperação da argila filtrante empregada na clarificação de óleo vegetal, empregando tratamento térmico seguido do tratamento químico ou viceversa. Esta comunicação foca apenas nos tratamentos isolados, térmico e químico, para numa posterior etapa serem acoplados. 


\section{MATERIAIS E MÉTODOS}

A terra clarificante (Terra Clarificante Tonsyl) a ser regenerada foi fornecida pela Bunge alimentos, situada no distrito industrial de SUAPE - PE. Além da argila filtrante utilizada no clareamento do óleo vegetal, foi fornecida uma amostra da argila in natura, que servirá como referência para o tratamento.

\subsection{Tratamento Térmico}

Para o tratamento térmico, foi montado um planejamento fatorial completo $2^{3}$ acrescido de ponto central, no qual foram estudadas como variáveis de entrada o tempo de contato, temperatura do tratamento e massa de terra e tendo como variável resposta a redução de massa (remoção do óleo). As amostras foram pré-tratadas a temperaturas de 40 e $105{ }^{\circ} \mathrm{C}$, para remoção da umidade e de voláteis. Desta forma foram realizados dois planejamentos experimentais, um para cada temperatura de prétratamento. As amostras foram colocadas em capsulas de porcelana de $255 \mathrm{~mL}$ e levadas à estufa para o pré-tratamento $\left(40\right.$ ou $\left.105^{\circ} \mathrm{C}\right)$ e em seguida à mufla (para o tratamento térmico conforme condições estabelecidas para o planejamento). Os experimentos foram realizados em duplicata com triplicata no ponto central. A Tabela 1 apresenta as variáveis com os níveis estudados. Após resfriar em dessecadores, as amostras foram novamente pesadas e calculadas as reduções de peso (remoção de óleo).

Tabela 1 - Variáveis e níveis utilizados no planejamento fatorial $2^{3}$ do tratamento térmico, após o prétratamento a $40{ }^{\circ} \mathrm{C}$ ou a $105^{\circ} \mathrm{C}$.

\begin{tabular}{|l|l|l|l|}
\hline Variável & -1 & 0 & +1 \\
\hline Temperatura $\left({ }^{\circ} \mathrm{C}\right)$ & 200 & 600 & 1000 \\
\hline Tempo $(\min )$ & 30 & 60 & 90 \\
\hline Massa $(\mathrm{g})$ & 5 & 15 & 40 \\
\hline
\end{tabular}

\subsection{Tratamento Químico}

Para a extração com solventes (Figura 1), testou-se álcool etílico P.A; álcool metílico P.A; ciclohexano P.A e tolueno P.A. Os experimentos foram realizados em Soxhlet. Tomando-se iguais quantidades de amostra, foi adicionado um volume de $150 \mathrm{~mL}$ de cada solvente (álcool etílico, álcool metílico, tolueno e ciclohexano) e seu respectivo balão de $250 \mathrm{~mL}$, baseando-se na solubilidade dos pigmentos nesses solventes, e segundo a temperatura de ebulição de cada um deles. Operações de até 12 horas de contato sólido-líquido foram realizadas. 


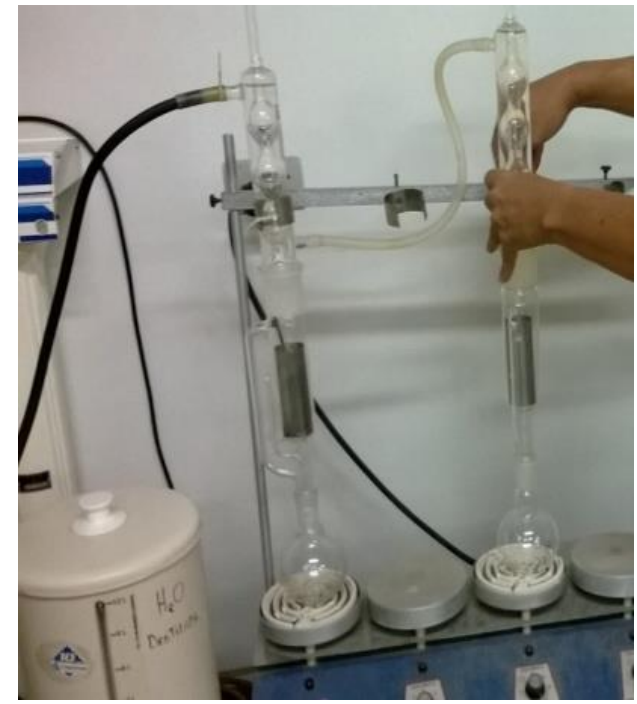

Figura 1 - arranjo experimental do tratamento químico.

Finalizada a extração retirou-se o cartucho (Cartuchos de papel de filtro quantitativo Whatman, diâmetro $185 \mathrm{~mm}$ ) encaminhando-o à estufa para secagem para a eliminação do solvente residual na amostra tratada. $\mathrm{O}$ solvente que contém os pigmentos é então, recuperado da solução por aquecimento, enquanto os pigmentos concentram-se no fundo do balão.

\section{RESULTADOS E DISCUSSÃO}

\subsection{Tratamento Térmico}

Inicialmente serão apresentados os resultados obtidos no planejamento experimental com o prétratamento a $105^{\circ} \mathrm{C}$. Como pode ser observado pelo Gráfico de Pareto (Figura 2a), entre as três variáveis independentes (de entrada), temperatura e massa foram as que apresentaram maior efeito significativo seguidas da interação massa-tempo. Ainda constatou-se, através da Figura 2a, o efeito positivo da temperatura, ou seja o seu aumento dentro dos níveis estudados favoreceu uma maior remoção.

$\mathrm{Na}$ superfície de resposta, apresentada na Figura $2 \mathrm{~b}$, observa-se graficamente o efeito da iteração entre o tempo e a massa durante o pré-tratamento a $105^{\circ} \mathrm{C}$, através da qual se constata que maiores remoções foram obtidas com menores quantidades de massa a um intervalo de tempo menor. 

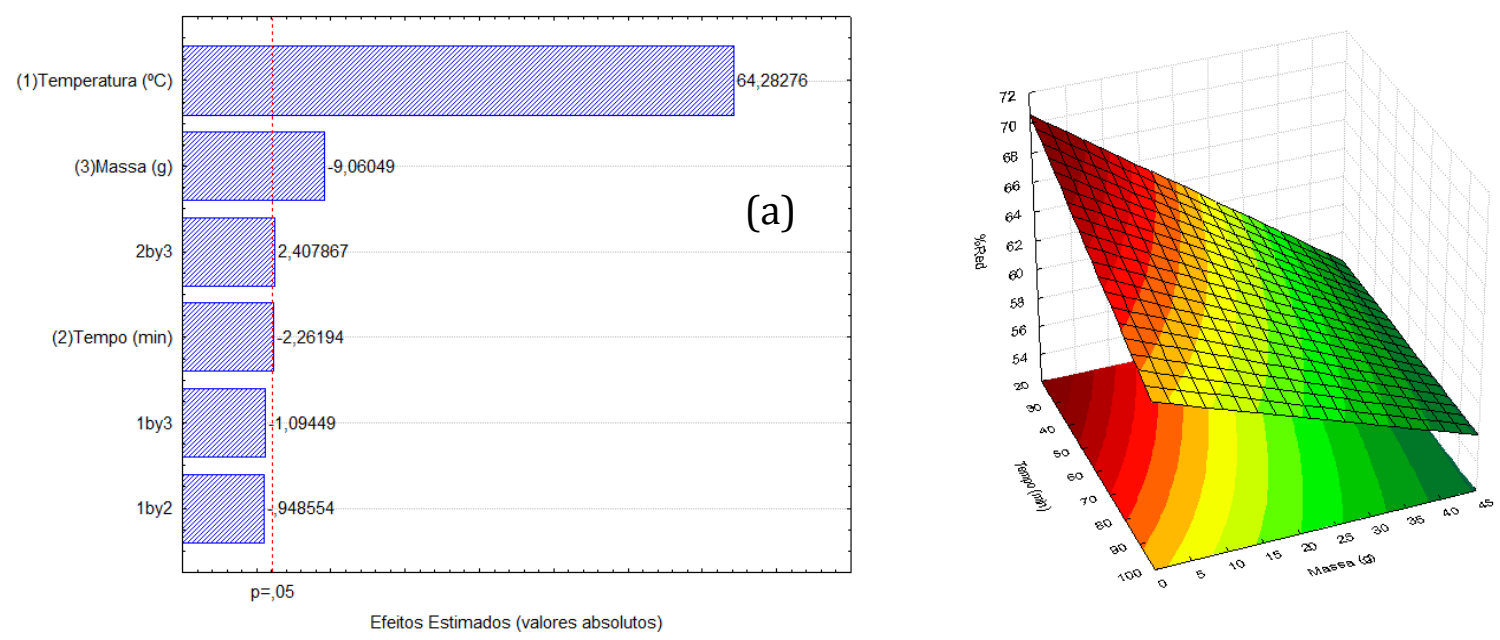

(b)

Figura 2 - Diagrama de Pareto (a) e superfície de resposta (b) para o pré-tratamento a $105{ }^{\circ} \mathrm{C}$ da terra clarificante

Através da Análise da variância (ANOVA) apresentada na Tabela 2, constata-se um bom ajuste dos dados experimentais, com o coeficiente de correlação $\left(\mathrm{R}^{2}\right)$ igual a 0,90 e variação máxima explicável de 99,72\%. Pode-se constatar ainda que o Fcal é maior que o Ftab, e a sua relação é maior que 5, logo, de acordo de Barros Neto et al. (2007) o modelo é significativo e preditivo. A Equação 1 apresenta o modelo estatístico com apenas as variáveis significativas.

Rend $(\%)=0,072 T-0,294 M+0,00197 t M$

na qual, $\mathrm{T}=$ Temperatura, $\mathrm{M}=$ Massa e $\mathrm{t}=$ tempo

Tabela 2 - Resultados da Anova para o pré-tratamento a $40{ }^{\circ} \mathrm{C}$ da terra clarificante

\begin{tabular}{|l|c|c|c|}
\hline \multicolumn{1}{|c|}{ Fonte de Variação } & Soma Quadrática & Números de Graus de Liberdade & Média Quadrática \\
\hline Regressão & 12406,6303 & 6 & 2067,7717 \\
\hline Resíduos & 1392,840664 & 13 & 107,1416 \\
\hline Falta de Ajuste & 1354,69 & 2 & 677,3439 \\
\hline Erro Puro & 38,15 & 13 & 2,9348 \\
\cline { 1 - 2 } Total & 13799,47 & 21 & $\mathbf{6 , 6 3}$ \\
\hline Ftabelado REG & $\mathbf{2 , 9 1}$ & Fc/Ftab & \\
\cline { 1 - 2 } Fcalculado & $\mathbf{1 9 , 3 0}$ & & \\
\cline { 1 - 2 } \%x. Explicada & 89,91 & & \\
\cline { 1 - 2 } \%Mx. Explicável & 99,72 & &
\end{tabular}


Já para o pré-tratamento a $40^{\circ} \mathrm{C}$, constatou-se pelo gráfico de Pareto (Figura 3a) que apenas a temperatura e a massa, como variáveis independentes, exerceram efeitos significativos. Todavia, assim como para o tratamento a $105^{\circ} \mathrm{C}$, a temperatura foi a varável que apresentou maior efeito e positivo e a massa apresentou efeito negativo, ou seja, quanto menor a massa melhor a eficiência do processo. Este fato está correlacionado à área exposta para o aquecimento mais direto e limitações de transferência de massa, como da saída do $\mathrm{CO}_{2}$. Pela superfície de resposta (Figura 3b) observa-se a discreta contribuição da massa em relação a temperatura, pois as linhas estão quase paralelas ao eixo da massa.
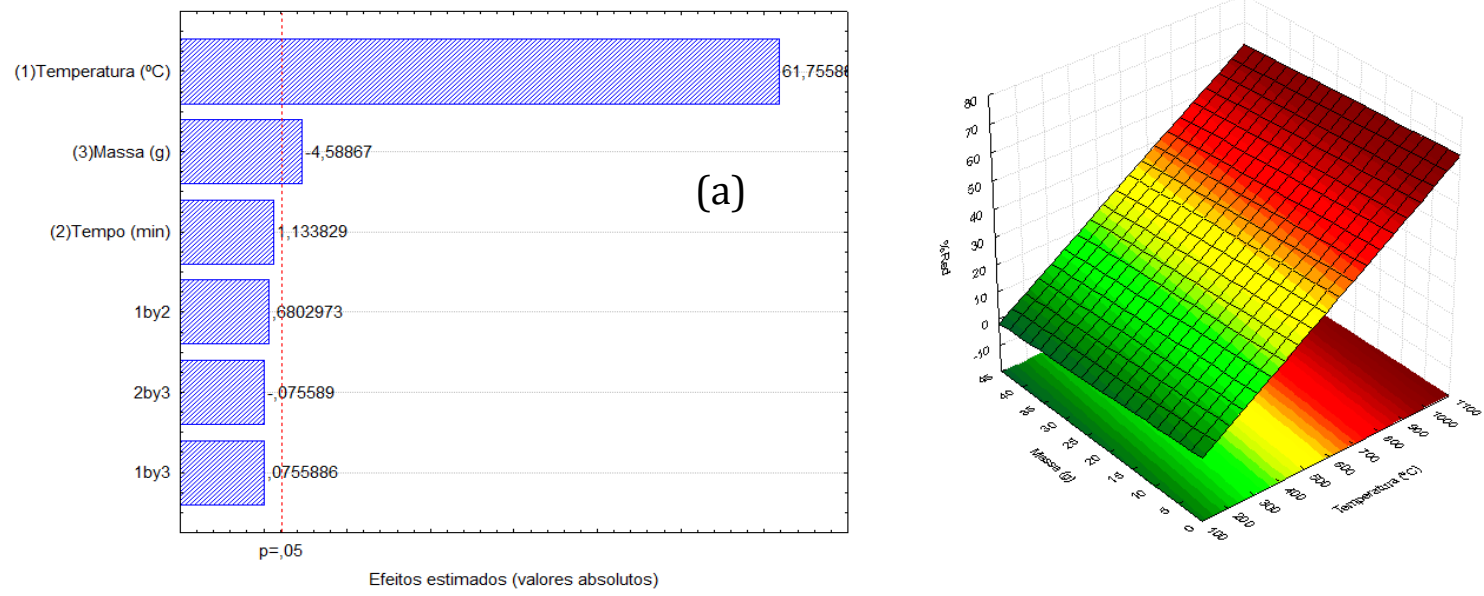

(b)

Figura 3 - Diagrama de Pareto (a) e superfície de resposta (b) para o pré-tratamento a $40{ }^{\circ} \mathrm{C}$ da terra clarificante

Em relação a avaliação estatística (ANOVA), apresentada na Tabela 3, verifica-se que o ajuste da correlação foi boa $\left(\mathrm{R}^{2}=0,88\right)$, com $99,70 \%$ de percentual máximo explicável e que o modelo apresentado na Equação 2, é estatisticamente significativo, ao se analisar o Fcal e o Ftab.

Tabela 3 - Resultados da Anova para o pré-tratamento a $40{ }^{\circ} \mathrm{C}$ da terra clarificante

\begin{tabular}{|c|c|c|c|}
\hline Fonte de Variação & Soma Quadrática & Números de Graus de Liberdade & Média Quadrática \\
\hline Regressão & 10491,90949 & 6 & 1748,6516 \\
\hline Resíduos & 1473,516018 & 13 & 113,3474 \\
\hline Falta de Ajuste & 1437,97 & 2 & 718,9825 \\
\hline Erro Puro & 35,55 & 13 & 2,7347 \\
\hline Total & 11965,43 & 21 & \\
\hline Ftabelado REG & 2,91 & & \\
\hline Fcalculado & 15,43 & $\mathrm{Fc} / \mathrm{Ftab}$ & 5,30 \\
\hline$\% \mathrm{Mx}$. Explicada & 87,69 & & \\
\hline$\% \mathrm{Mx}$. Explicável & 99,70 & & \\
\hline $\mathrm{R}^{2}$ & $\mathbf{0 , 8 8}$ & & \\
\hline
\end{tabular}


Rend $(\%)=0,062 \mathrm{~T}-0,105 \mathrm{M}$

na qual, $\mathrm{T}=$ Temperatura e $\mathrm{M}=$ Massa

\subsection{Dessorção com solventes}

Em relação ao experimento de dessorção com os solventes, obteve-se um rendimento médio de $22,5 \%$ para o tolueno, de 31,5\% para o álcool etílico, de 27,75\% para o álcool metílico e de 17,85 para o ciclohexano.

\section{CONCLUSÕES}

Tendo em vista os resultados obtidos, observou-se que o melhor tratamento térmico foi a $1000^{\circ} \mathrm{C}$ durante 30 minutos com 5 gramas de argila filtrante, com um pré-tratamento a $105^{\circ} \mathrm{C}$. Em relação aos solventes estudados, o etanol foi o que apresentou melhor remoção (31,5\%). Desta forma, será estudado agora um pré-tratamento com álcool etílico, passando por uma estufa a $105^{\circ} \mathrm{C}$ e seguindo para a mufla, com um novo planejamento. Análises de Difração de raios X estão sendo realizadas para se avaliar o efeito do aquecimento na estrutura da terra clarificante. Os modelos obtidos para ambas temperaturas de pré-tratamento permitem obter percentuais de remoção em função das condições operacionais. Está sendo realizada a caracterização dos materiais por difração de raios X e área superficial e volume poroso para se identificar alterações na composição e estrutura das argilas após e tratamento.

\section{AGRADECIMENTOS}

Os autores agradecem ao CNPq, CAPES, FACEPE pelo apoio financeiro e bolsas concedidas, assim como ao ITEP e CETENE pela realização das análises e caracterização dos materiais.

\section{REFERENCIAS}

ABIOVE - Associação Brasileira das Indústrias de Óleos Vegetais - Exportações do Complexo Soja Março/2014 - disponível em http://www.abiove.org.br/site/index.php?page= estatistica\&area =NC0yLTE $=$ visualizado em 111/04/2014

BARROS NETO, B.; SCARMINIO, I. S.; BRUNS, R. E. Como fazer experimentos: pesquisa e desenvolvimento na ciência e na indústria. v.1, 4.ed. Coleção Livros-Textos, Campinas: UNICAMP, 480 p. 2007.

CARDELlO, H.M.A.B., BORGHI, A.B.M.P., VILA, M.M.D.C., GONÇALVES, L.A.G., Clarificação convencional de óleo de sementes de algodão (Gossipium hirsutum). Alim. Nutri. v. 6, p. 77-87, 1995. 
GAROFALO, M.N. Regeneração de Argilas Clarificantes da Indústria de Refino de Óleos Vegetais. Dissertação de Mestrado do Programa de Pós-Graduação em Engenharia Química da Universidade Federal de Santa Catarina. 76p. 2011.

GÜLLER, Ç., TUNÇ, F., Chlorophyll Adsorption on Acid-actived Clay, J. Am. Oil Chem. Soc., v. 69, p. 948-950, 1992.

GONZÁlEZ-TOVAR, L. A. NORIEGA-RODRÍGUEZ, J. A. ORTEGA-GARCÍA, J. GÁMEZMEZA, N., MEDINA-JUÁREZ, L. A. Cinética de adsorción de pigmentos, peróxidos y tocoferoles durante el processo de blanqueo del aceite de soja. Grasas y Aceites. v. 56, n. 4, p. 324-327, 2005.

LIMA, N.S. Recuperação de rejeitos da clarificação de óleos vegetais por dessorção / extração. Dissertação de Mestrado. Programa de Pós-Graduação em Engenharia Química da Universidade Federal de Pernambuco. 95p. 2003.

MEZITI, C., BOUKERROUI, A. Regeneration of a solid waste from an edible oil refinery. Ceramics International. v.37, p.1953-1957, 2011.

PATTERSON, H.B.W. Bleaching and Purifying Fats and Oils: Theory and Practice, American Oil Chemists Society Press, 242p. 1992.

O'BRIEN, R.D. FARR, W.E., WAN, P.J. Introduction to Fats and Oils Technology. AOCS Press, 618 p. 2000.

SABAH, E., INAR, M.C, ELIK, M.S.C. Decolorization of vegetable oils: Adsorption mechanism of bcarotene on acid-activated sepiolite. Food Chemistry. v. 100, p.1661-1668, 2007.

SILVA, S.M., SAMPAIO, K.A., CERIANI, R. VERHÉ, R., STEVENS, C., GREYT, W.D., MEIRELLES, A.J.A. Adsorption of carotenes and phosphorus from palm oil onto acid activated bleaching earth: Equilibrium, kinetics and thermodynamics. Journal of Food Engineering. v. 118, p. 341-349, 2013.

SONNTAG, N. O. V., FORMO, M. W., JUNGERMANN E., NORRIS, F. A., Bailey's industrial oil and fat products, v.1, 4a ed., John Wiley \& Sons, 1979.

TEIXEIRA-NETO, E., TEIXEIRA-NETO, A.A., Modificação química de argilas: desafios científicos e tecnológicos para obtenção de novos produtos com maior valor agregado. Quim. Nova, v. 32, n. 3, p. 809-817, 2009.

USDA - United States Department of Agriculture. Economic Research Service. Table 47: World vegetable oils supply and distribution, 2009/10-2013/14. disponível em: http://www.ers.usda.gov/data-products/oil-crops-yearbook.aspx\#.U0gX6lcjaMQ, acessado em 11/04/2014. 